over capital gains tax. I doubt if many accountants inject their own haemorrhoids and likewise I would advise you not to attempt your own accounts. It is worth talking to your accountant well before the first tax demand appears as a certain amount of forward planning is needed. You may for instance need to set the end of your accounting year at the end of April, allowing an extra year's breathing space before the first tax is due. To someone brought up on PAYE the need to write cheques for the Inland Revenue on fees long since spent may come as an embarrassment. Thus from the start it is prudent to set aside, say, $25 \%$ of your private earnings in anticipation of a juicy tax demand. Although schedule $\mathrm{D}$ is more generous with allowances than the schedule E of PAYE, the costs of setting up in practice are by no means all tax deductible, so don't rush out and buy a new car.

New consultants may be anxious about what fees they should charge patients. This need not be a source of anxiety as in each geographical area there are fairly standard fees for new and for follow up consultations and the insurance companies issue lists categorising operations into minor, intermediate, major, and major plus types. In addition they settle the amount that they will reimburse for an operation in any category. There are ill concealed murmurs of dissatisfaction about the levels of these fees and their unbalanced nature favouring certain specialties. Nevertheless, insured patients reasonably expect to be charged what the insurance company specifies, and if you intend to charge more than that you ought to tell the patient in advance.

\section{Conclusion}

However small it might be, most people find private practice is instructive, stimulating, and rewarding-so much so that its enticements may be considerable - and it will thus do no harm to conclude with a warning about abuses of private practice that can too easily be slipped into. These include overtreating, overcharging, and overvaluing your own skills so that you are tempted to treat in private practice those conditions that you would refer to your colleague in the context of the health service. Lastly, remember to be punctilious about your commitments in the NHS. Don't let an enthusiasm for private practice nibble into your NHS time. Your junior staff may not get any formal education in private practice but watching you will be their informal education and it should be correct.

\title{
Impotence: treatment by autoinjection of vasoactive drugs
}

\author{
GORDON WILLIAMS, M J MULCAHY, E A KIELY
}

\begin{abstract}
One hundred and twenty five men with psychogenic or organic impotence used autoinjection of the penile corpora cavernosa with either papaverine or papaverine and phentolamine for a mean period of 10.5 months (range 1-24 months) to achieve penile erection for sexual intercourse. Prolonged (over four hours) painless erection resulted from $\mathbf{3 4}$ of the $\mathbf{3 5 1 3}$ self administered injections.
\end{abstract}

This seems to be a highly effective approach to treating impotence irrespective of the aetiology.

\section{Introduction}

Advances in the investigation, diagnosis, and treatment of impotence $^{1}$ have led to more and more men being referred for treatment. Half of those who are referred for treatment have a predominantly organic cause. ${ }^{2}$ Men who have venous leaks (5-10\%) can be treated by surgery. ${ }^{3}$ Penile revascularisation procedures for men with arteriogenic impotence are available in only a few centres, and results are generally poor..$^{46}$ Endocrine treatment for a low serum testosterone or high prolactin concentration is rarely effective. ${ }^{7}$ Few of the remaining patients will be offered or can afford a penile implant. Those with psychogenic impotence respond poorly to psychotherapy. ${ }^{8}$

Hammersmith Hospital, London W12 0HS

GORDON WILLIAMS, MS, FRCS, consultant urologist

M J MULCAHY, MB, FRCSI, urological registrar

E A KIELY, MB, FRCSI, urological registrar

Correspondence to: Mr G Williams, Department of Surgery, Royal Postgraduate Medical School, Hammersmith Hospital, Ducane Road, London W12 0HS.
Intracavernosal injections of papaverine or papaverine and phentolamine are both diagnostic and therapeutic ${ }^{19}$ : diagnostic because the failure to achieve an erection when arterial inflow is normal suggests venous leakage, and therapeutic because patients with psychogenic impotence often regain spontaneous erections. ${ }^{11}$ Most of these patients or their partners can be easily taught to administer the injection, allowing the time of the erection to be varied to suit them and their partners. ${ }^{10}$

We report the results of treatment of 125 men with impotence of various aetiologies who practised self injection.

\section{Patients and methods}

Altogether 181 men who were referred to the male sexual dysfunction clinic underwent a full clinical history and examination. Their penile brachial index was determined using a $10 \mathrm{mHz}$ Doppler probe as a measure of arterial inflow, 1 and liver function tests were performed and blood glucose and plasma lipid concentrations measured. Serum testosterone and prolactin concentrations were also measured.

Patients who show a poor erectile response to intracavernosal papaverine and phentolamine and have a penile brachial index of $>0.7$ have venous leaks (unpublished results). Sixteen such patients were excluded from this study, as were three patients with abnormal results from liver function tests and 17 whose potency returned after their first pharmacologically induced penile erection. Of the remaining 145 patients, 125 aged from 27 to 79 (mean 49) entered the autoinjection programme; 109 had regular partners. Sixty two patients had diabetes mellitus, of whom 46 were insulin dependent. Of these 62,18 had either a peripheral or an autonomic neuropathy, and 21 had an abnormally low penile brachial index of $<0.6$. No vascular or neurological abnormality was found in the remaining 23 . A penile arteriopathy was suggested in a further 25 non-diabetic patients who had a penile brachial index of $<0.6$. Fourteen patients had neurogenic impotence: four as the result of spinal cord injuries and three after pelvic surgery, six had multiple sclerosis, and one had Guillain-Barré syndrome. In the remaining 
23 patients no organic cause could be found, and they were presumed to have predominantly psychogenic impotence. No patient had abnormal serum testosterone or prolactin concentrations.

Erections were induced by an intracavernosal injection of either a combination of papaverine $30 \mathrm{mg}$ and phentolamine $1 \mathrm{mg}$, prepared by the hospital pharmacy, or papaverine alone in a dose ranging from 7.5 to $30 \mathrm{mg}$. Based on results of our previous studies all patients with a diagnosis of neurogenic impotence or psychogenic impotence received $7.5 \mathrm{mg}$ papaverine alone for their initial erection, and the dose was then adjusted to the patient's response. ${ }^{9}$

After at least three visits most patients were able to draw up and give themselves the injection. They were told not to inject more than twice a week and to report immediately to the hospital, where a 24 hour specialist service was available, if complications arose. They were told that the dose might have to be reduced while injecting in their homes and were asked to try to achieve an erection by normal means before autoinjection to determine the frequency of onset of spontaneous erections. They were also told to return to the hospital if an erection lasted more than four hours. A questionnaire was completed by each patient after each injection. Patients were seen at four weeks and then at three month intervals, when liver function tests and penile examination were carried out. All injections were given through a 25 or 27 gauge needle.

\section{Results}

The patients had been followed up for a mean of 10 months (range one to 24 months) at the time of writing. A total of 3860 injections had been self administered, resulting in 3513 satisfactory erections. The median number of injections given was 27 . Twenty patients stopped self injecting, five lost their partners, potency returned in 11 , and four were lost to follow up. The 11 who regained their potency were classified as having predominantly psychogenic impotence, though three were also diabetic. Fourteen patients claimed to have more frequent and stronger nocturnal erections. The patients who had psychogenic impotence injected less often after six weeks' treatment (mean 0.9 injections a month), whereas those with an organic aetiology continued to inject regularly (mean 3.5 injections a month). The dosage of the drugs was reduced in 26 patients to produce an erection of shorter duration. Eleven had a pure neurogenic aetiology, eight diabetes (four of whom had a neuropathy), and seven psychogenic impotence.

No patient developed either local or systemic infection, fibrosis of the corpora, or alteration in results of liver function tests. Minor bruising occurred in 27 patients early in their treatment, and 18 had pain at the site of injection. One patient inadvertently injected the corpora spongiosum and passed blood. Prolonged erections occurred after 34 of the 3513 pharmacologically induced erections: eight patients had a neuropathy and 26 psychogenic impotence. The erections lasted between six and 23 hours in five patients despite instructions to return to hospital after four hours. Thirty patients required decompression using the technique described by Brindley. ${ }^{12}$ In four cases the erections subsided spontaneously after eight, nine, 10 , and 13 hours. All patients continued to self inject with a reduced dose. One patient required decompression after injecting a double dose.

\section{Discussion}

Successful treatment of impotence has not been available to most men. Pharmacologically induced erections in which a doctor gives the injection have been of long term benefit in some patients with psychogenic impotence. ${ }^{1910}$ Patients who are dependent long term on this treatment, however, are best served by self injection. This is a highly acceptable alternative to a penile implant prosthesis because there are no complications of surgery, ${ }^{1314}$ which may occur in up to $40 \%$ of cases, and it allows the possibility of a return of spontaneous erections. Vacuum condoms are less invasive than injections, but long term results are still awaited (unpublished results).

Autoinjection was readily accepted by most of the patients, with
3513 erections resulting from 3860 injections. This shows that the patient can learn and master the technique of self injection. It is also of value for patients with associated premature ejaculation because the erection remained even after ejaculation. Potency returned to eight of 24 patients in whom no organic cause could be found and to three diabetics who had no evidence of arterial or neurogenic complications. This is attributed to reduced anxiety about performance because of self injection. ${ }^{9}$ is The improvement is gradual; three patients required intermittent injections to boost their erections. Patients with no obvious organic abnormality are able to reduce the frequency of injection or to stop injecting. Twenty six of the 125 patients required a reduction in the original dose, and the dose never exceeded $30 \mathrm{mg}$ papaverine and $1 \mathrm{mg}$ phentolamine, so tolerance to the drugs did not occur. These findings agree with those of Zorgniotti and Lefleur. ${ }^{10}$

Forty six patients were insulin dependent diabetics. Though these patients are more susceptible to sepsis, this was not observed in any patient. Sidi et al reported (American Urological Association, May 1987) that seven of 300 patients developed localised areas of cavernosal fibrosis immediately adjacent to the corpora, which was perhaps related to poor injection technique. Drugs injected into the corporal fascia caused a localised haematoma. This complication was not seen in our series. The most inconvenient complication in our series was prolonged erections. Despite warnings five patients delayed returning to hospital. It is essential that facilities for decompression in a specialist unit are available at all times. Decompression was carried out easily and without side effects. Haematoma and bruising resulting from decompression were more severe than those produced by the initial intracavernosal injection. Prolonged erections occurred only in patients with neurogenic or psychogenic impotence. These patients must be monitored carefully to allow titration of the necessary dose. The use of papaverine has been associated with abnormal results of liver function tests, but this was not seen in our series with a follow up of up to two years. Longer follow up is necessary to establish the safety of autoinjection. More extensive long term studies in the treatment of impotence by autoinjection of vasoactive compounds are warranted.

\section{References}

1 Virag R, Frydman D, Legman $M$, Virag $H$. Intracavernous injection of papaverine as a diagnostic and therapeutic method in erectile failure. Angiology 1984;35:79-87.

2 Smith AD. Causes and classification of impotence. Urol Clin North Am 1981;8:79-89.

3 Wespes E, Schulman CC. Venous leakage: surgical treatment of a curable cause of impotence. f Urol 1985;133:796-8.

4 Crespo E, Soltanik E, Bove D, Farrell G. Treatment of vasculogenic sexual impotence by revascularizing cavernous and/or dorsal arteries using microvascular techniques. Urology 1982;20:271-5.

5 MacDougal WS, Jeffrey RF. Microscopic penile revascularization. $f$ Urol 1983;128:517-21.

6 Bennett AH, Rivard DJ, Blanc P, Moran M. Reconstructive surgery for vasculogenic impotence. J Urol 1986;136:599-601

7 Zorgniotti AW. Practical diagnostic screening for impotence. Urology 1984;23:98-102.

8 Bancroft J. Helping people with sexual problems. In: Human sexuality and its problems. Edinburgh: Churchill Livingstone, 1983:240-317.

9 Kiely EA, Williams G, Goldie L. An assessment of the immediate and long term effects of pharmacologically induced penile erections in the treatment of psychogenic and organic impotence. Br f Urol 1987;59:164-71.

10 Zorgniotti AW, Lefleur RS. Auto-injection of the corpus cavernosum with vasoactive drug combination for vasculogenic impotence. 7 Urol 1985;133:39-41.

11 Velcek D, Sniderman KW, Vaughan ED Jr, Sos TA, Muecke EC. Penile flow index utilizing a Doppler pulse wave analysis to identify penile vascular insufficiency. $\mathcal{F}$ Urol 1980;123:669-73. 2 Brindley GS. New treatment for priapism. Lancet 1984;ii:220-1.

13 Apte SM, Gregory JG, Purcell MH. The inflatable penile prosthesis, reoperation and patient Apte SM, Gregory JG, Purcell MH. The inflatable penile prosthesis, reoperation and patient
satisfaction: a comparison of statistics obtained from patient record review with statistics satisfaction: a comparison of statistics obtained from patient recor
obtained from intensive follow up search. $\mathcal{J}$ Urol 1984;131:894-5.

14 Kaufman JJ, Linder A, Raz S. Complications of penile prosthesis surgery for impotence. $\mathcal{J} \mathrm{Urol}$ 1982;126:1192-4.

15 Osborne D. Psychological aspects of male sexual dysfunction. Urol Clin North Am 1981;8:135-42.

(Accepted 11 fune 1987) 